\title{
ESTUDIO PRELIMINAR DE EVALUACIÓN DE LA CREATIVIDAD ARTÍSTICA MEDIANTE EL ANÁLISIS DE SEÑALES ELECTROENCEFALOGRÁFICAS
}

\author{
Mario Ortiz \\ Dpto. Ing. Mecánica y Energía. Brain-Machine Interface Systems Lab, Universidad Miguel Hernández de \\ Elche, Av. Universidad S/N Ed. Innova. 03202 Elche, España, mortiz@umh.es \\ Marisol Rodríguez-Ugarte, Eduardo Iáñez, José M. Azorín \\ Dpto. Ing. de Sistemas y Automática. Brain-Machine Interface Systems Lab, Universidad Miguel Hernández \\ de Elche, Av. Universidad S/N Ed. Innova. 03202 Elche, España, maria.rodriguezu@umh.es, \\ eianez@umh.es,jm.azorin@umh.es
}

\begin{abstract}
Resumen
Para evaluar la creatividad es habitual el uso de tests de creatividad, tales como el test de pensamiento creativo de Torrance. Su empleo está extendido en el ámbito de la educación e incluso en el mundo empresarial. No obstante, por la propia naturaleza de estas pruebas resulta necesaria la supervisión por parte de diversos expertos de las tareas realizadas, no dejando de tener esta evaluación una parte subjetiva. Mediante nuestra investigación, pretendemos plantear las bases para poder evaluar de forma alternativa la creatividad de un sujeto, en base a la evaluación de las señales electroencefalográficas durante un proceso de pensamiento y acción creativa. Este artículo muestra los procedimientos a llevar a cabo durante los tests de creatividad y el tratamiento de la señal eléctrica a realizar tras los mismos. Todo ello, con el fin de definir en futuras investigaciones un índice objetivo de creatividad.
\end{abstract}

Palabras Clave: Interfaz cerebro-computador, test de creatividad, señales electroencefalográficas, creatividad, análisis en tiempo-frecuencia.

\section{INTRODUCCIÓN}

El concepto de creatividad y pensamiento creativo resulta complejo de definir. Su definición más básica sería la de generar nuevas ideas o conceptos a partir de la asociación de ideas y conceptos conocidos, con el fin de producir soluciones originales. En el caso de la creatividad asociada al arte, se añade el elemento adicional de la estética que acompaña a la creatividad artística. Aunque en nuestro estudio se estudiará la creatividad asociada al arte pictórico, este concepto puede ser extendido a otros aspectos de la vida como pueden ser ciencia, economía, diseño, etc.
Para evaluar la capacidad de pensamiento creativo, lo habitual es el uso de tests de creatividad. El más extendido es el test de pensamiento creativo de Torrance [14]. Los resultados de estos tests tienen relevancia no sólo en el mundo de la educación, para detectar niños con capacidades especiales, sino también empresarial, con el fin de seleccionar personas especialmente creativas que puedan aportar soluciones innovadoras a los problemas que surgen en el día a día de una compañía. No obstante, la propia naturaleza multidimensional del pensamiento creativo hace que, para una correcta evaluación, sea necesario realizar diversos tipos de tests o incluso apoyarse en otros indicadores como puedan ser: resultados académicos, recomendaciones, tests de coeficiente intelectual, etc. [6]. Adicionalmente, el proceso de evaluación requiere de una evaluación por parte de varios expertos, que no deja de ser subjetiva, aunque se intente reglar mediante unos criterios de evaluación predefinidos [5].

Todo proceso creativo-artístico consta de dos fases: improvisación inicial, donde se lleva a cabo la generación espontánea de las ideas a realizar, seguido por una fase de reevaluación y revisión [4]. En los últimos años se está intentando comprender la relación existente entre el cerebro y las tareas de carácter creativo-artístico. Sin embargo, aunque algunos estudios basados en resonancia magnética funcional (fMRI) sostienen que, por ejemplo la improvisación de un músico de jazz tiene relación con un patrón de actividad desasociada en el cortex prefrontal [8], o que la improvisación de un cantante de rap provoca una actividad desasociada en el cortex medio y en el cortex prefrontal dorsolateral [9] la relación es de mayor complejidad. De hecho, el estudio realizado por Dietrich et al. [3] analiza mediante diversas técnicas de registro la actividad cerebral, entre ellas electroencefalografía (EEG), llegando a la conclusión de que la creatividad como proceso de actividad cerebral, no parece depender de 
un único proceso mental, o de una única región cerebral, no tiene porqué tener relación con la atención desfocalizada, con la baja atención o incluso con la sincronización alfa, como se hipotetiza. Por ello para que sea posible analizar la creatividad en el cerebro, ésta debe ser subdividida en diferentes tipos que puedan ser asociados a procesos neurocognitivos específicos.

Adicionalmente, es preciso llevar una baremación basada en la actuación de diversos sujetos de pruebas con el fin de llevar a cabo una correlación entre la actividad creativa realizada y las señales electroencefalográficas obtenidas, con objeto de poder obtener una clasificación de la creatividad.

El artículo consta de las siguientes secciones:

- En la sección 2 se lleva a cabo una descripción de los equipos y materiales utilizados para la adquisición de las señales EEG durante las pruebas de creatividad artística.

- En la sección 3 se lleva a cabo una descripción del protocolo de pruebas.

- En la sección 4 se describe los procedimientos de análisis de señal a realizar una vez han sido adquiridas.

- En la sección 5 se muestra los resultados obtenidos una vez han sido tratadas las señales para un sujeto.

- En la sección 6 se muestran las conclusiones y las futuras acciones a seguir para completar la investigación.

\section{EQUIPOS Y MATERIALES}

\subsection{Interfaz cerebro-computador}

Un interfaz cerebro-computador (BCI) permite capturar las señales EEG de un sujeto con el fin de procesarlas y traducirlas en un comando que pueda ser interpretado por un computador. En el caso de esta investigación, se utiliza el BCI como medio para obtener una medida de la actividad cerebral del sujeto durante la realización de acciones creativoartísticas. Una vez capturadas, las señales son filtradas y procesadas para extraer la características a correlacionar con la actividad creativa.

El registro de datos se realizó mediante un dispositivo de la marca Brain Products $\mathrm{GmbH}$ (Alemania), compuesto por 31 electrodos para la adquisición de datos, situándose el electrodo de referencia en el lóbulo de la oreja derecha. La figura 1 muestra los electrodos utilizados para la medición de las señales EEG.

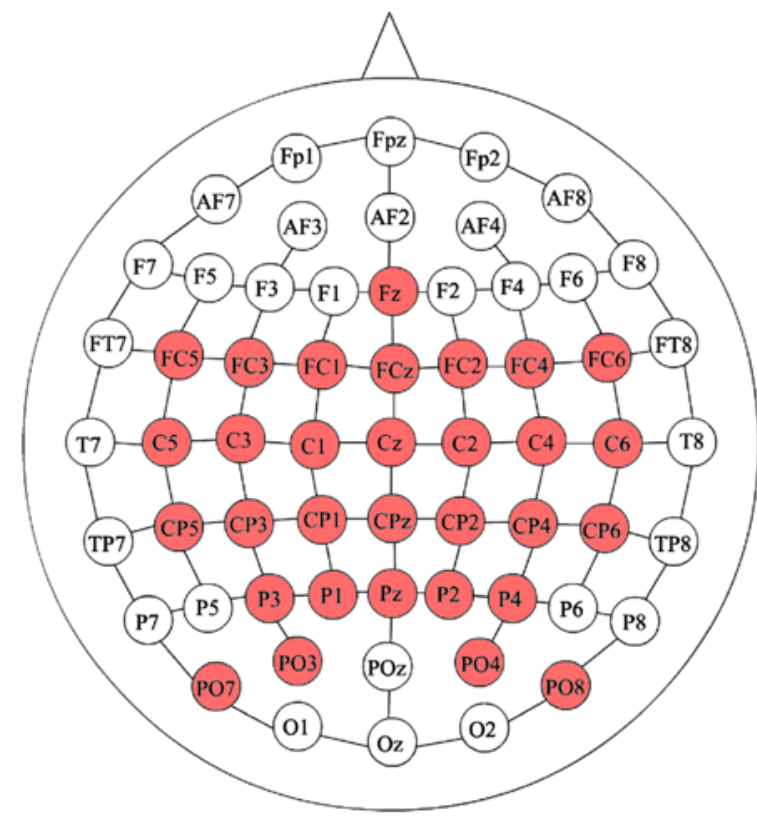

Figura 1: Electrodos utilizados para el registro de las señales EEG.

Los electrodos fueron posicionados con la ayuda de un gorro actiCAP del mismo fabricante. El registro de las señales se envió al ordenador por cable mediante un amplificador actiCHamp a una frecuencia de muestreo de $500 \mathrm{~Hz}$, actualizándose la ventana de medición cada 100 muestras (0.2 s). El software de registro fue el Brain Vision de Brain Products GmbH, transmitiéndose los datos a Matlab para su posterior procesamiento.

\subsection{Material artístico}

Con el fin de evaluar la capacidad creativo-artística de los sujetos de pruebas, diverso material artístico fue utilizado. Este consistió en pinceles, acuarela en tubo de diversos colores, lápices acuarelables, papel de dibujo y láminas de alta resolución de obras artísticas.

\section{PROTOCOLO DE PRUEBAS}

El estudio se realiza en acuerdo con las recomendaciones del comité de ética de la Universidad Miguel Hernández de Elche, obteniéndose consentimiento firmado por todos los sujetos de experimentación de acuerdo con la Declaración de Helsinki.

Debido a que la actividad cerebral durante un proceso creativo no depende de un solo proceso mental [3] el protocolo de pruebas intenta acotar el proceso creativo a la actividad y contemplación pictórica. Para ello, el proceso se realiza en 6 etapas diferentes. Todas las pruebas se inician con un pitido y finalizan con un doble pitido, despreciándose los 2 
segundos iniciales para evitar potenciales evocados por el estímulo sonoro.

\subsection{P1: Improvisación}

Para evaluar la actividad creativa durante el proceso de improvisación artística se le muestra al sujeto una lámina con manchas de acuarela en color negro. El sujeto tiene previamente los ojos cerrados y cuando suena un pitido los abre para contemplar la lámina durante 17 segundos. Durante esta prueba el sujeto se concentra en imaginar los colores y formas que su mente crea a partir del estímulo abstracto de la lámina. La figura 2 muestra la lámina utilizada para esta prueba.

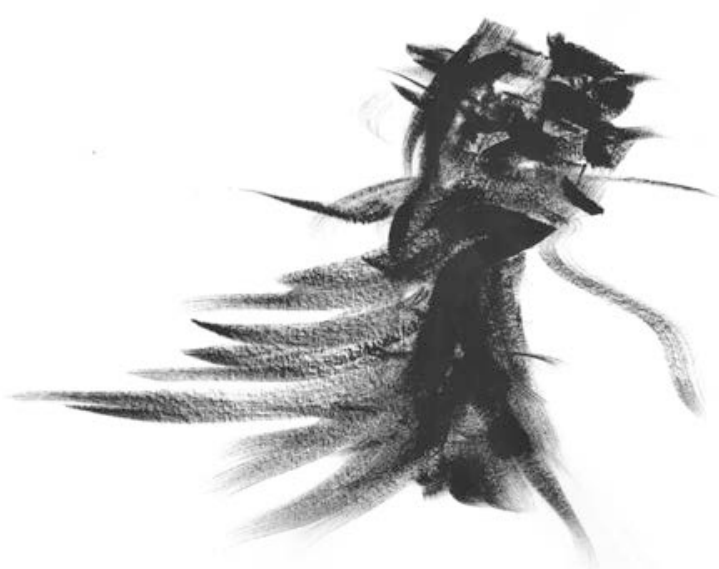

Figura 2: Lámina utilizada durante P1. Mediante ella se intenta dar un estímulo inicial similar a los sujetos con el fin de evaluar la actividad cerebral durante el proceso de imaginación e interpretación creativa de la imagen abstracta mostrada.

\subsection{P2: Actividad creativa 1}

Durante la siguiente prueba el sujeto plasma la imagen que improvisó su mente durante P1. Los sujetos preseleccionan los colores de acuarela en tubo que desean utilizar, comenzando la prueba una vez que está el material preparado. Durante la prueba, que dura 5 minutos más los 2 segundos iniciales, el sujeto es libre de modificar su idea original si durante el proceso creativo pictórico desea mejorarla. La figura 3 muestra un sujeto durante el inicio de esta prueba.

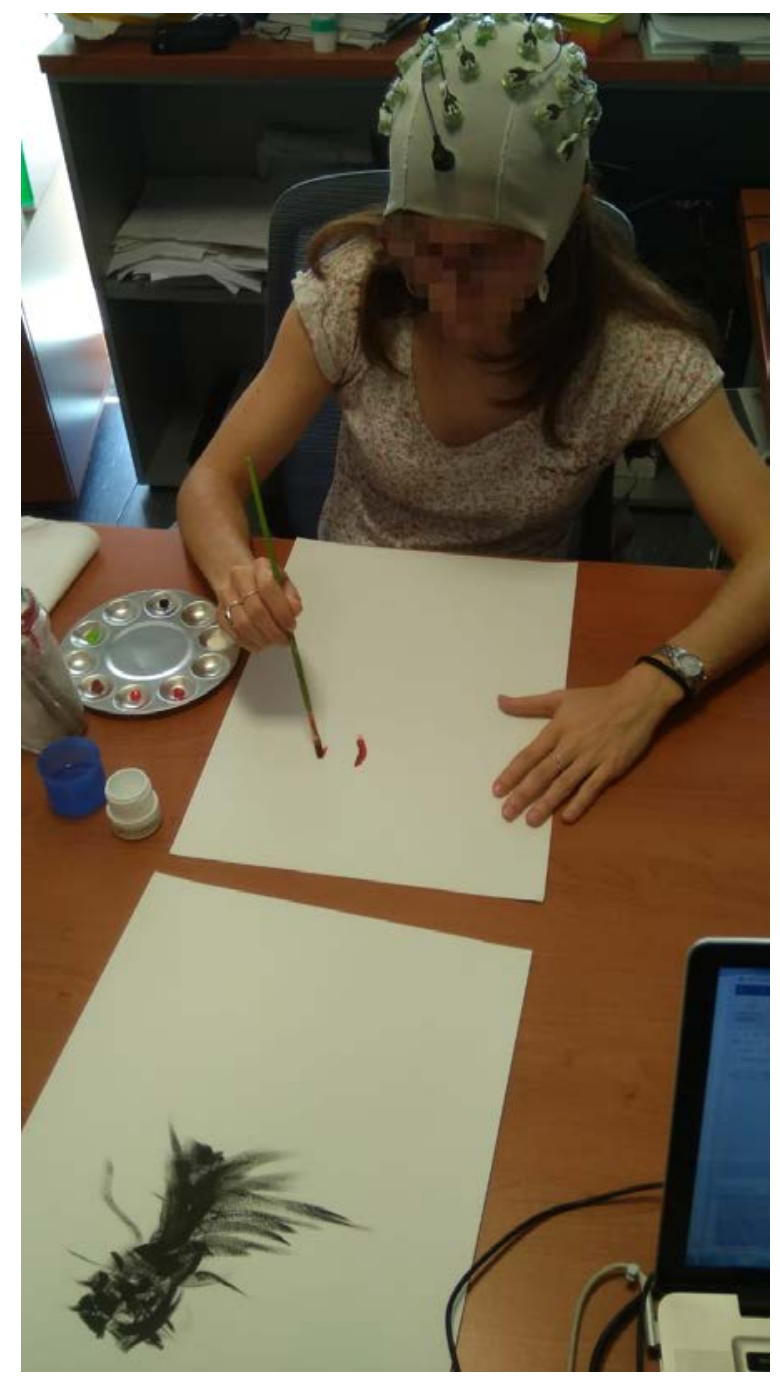

Figura 3: Desarrollo de la actividad creativa pictórica a partir de la lámina abstracta.

\subsection{P3: Improvisación asociada a la creación de una historia}

Con base en el test de Torrance [14], se le muestra durante 17 segundos una imagen sencilla formada por dos líneas en paralelo. A partir de ellas el sujeto de pruebas debe imaginar durante ese tiempo un dibujo del que formen parte esas líneas y un título para la obra que, de forma original, resuma lo que quiere representar.

\subsection{P4: Actividad creativa 2}

Al igual que en $\mathrm{P} 2$, durante 5 minutos el sujeto de pruebas lleva a cabo el desempeño de la actividad creativa imaginada durante la prueba previa. En este caso, y para intentar desagregar la técnica pictórica de la parte creativa, se utilizan lápices de acuarela, facilitando el uso a sujetos no habituados al uso de pinceles. La figura 4 muestra un sujeto llevando a cabo esta tarea 


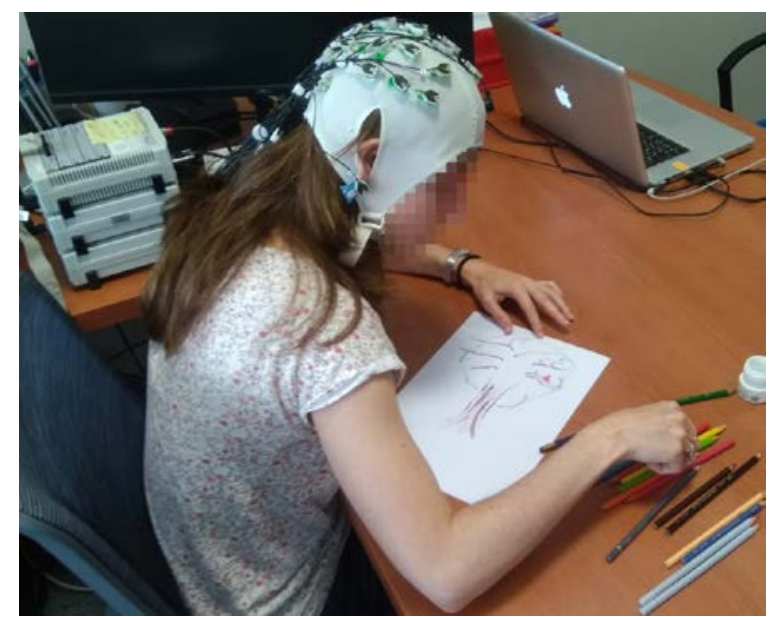

Figura 4: Desarrollo de la actividad creativa pictórica a partir de una figura simple de dos líneas.

\subsection{P5: Contemplación pictórica: Neuroestética}

Con el fin de obtener patrones de referencia, durante P5 se analiza la actividad cerebral del sujeto cuando contempla una obra artística. Para ello se le muestra durante 17 s una obra artística. La figura 5 muestra este proceso.

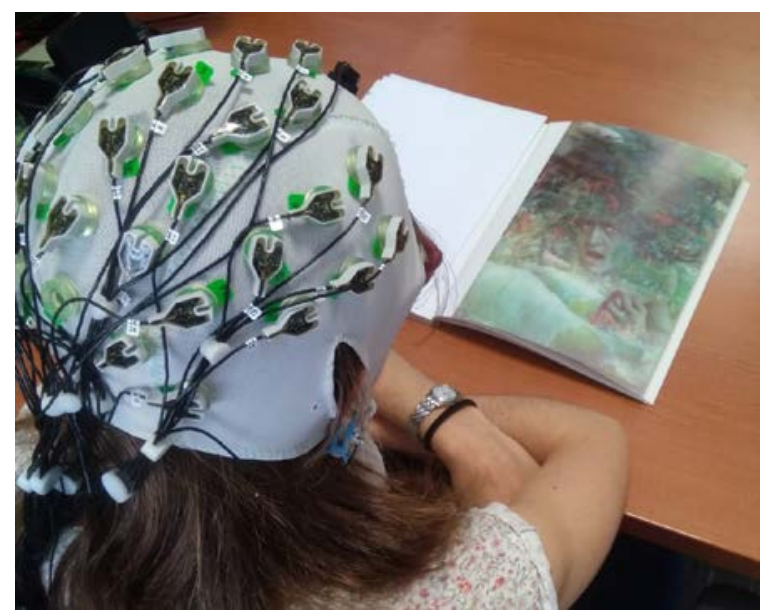

Figura 5: Contemplación de la obra seleccionada para P5: Medusa de Lucien Lévy-Dhurmer.

Con esta prueba se intenta evaluar la actividad cerebral durante la contemplación de una obra artística (neuroestética), objeto de actual investigación por sí misma como muestran diversos artículos [1,7].

\subsection{P6: Relax}

La última de las pruebas busca obtener la actividad del sujeto cuando deja su mente en blanco ajeno a cualquier tipo de pensamiento o acción. El sujeto ha de mantener los ojos abiertos durante el proceso. Esta parte dura igualmente 17 segundos.

\section{4 \\ TRATAMIENTO Y ANÁLISIS DE LOS DATOS}

El análisis de los datos conlleva diversas etapas. En primer lugar, se lleva a cabo un pretratamiento con el fin de mejorar la información obtenida por cada electrodo. Una vez filtrada esa información, los datos son normalizados con el fin de que la información pueda ser comparada en un mismo orden de magnitud para los distintos sujetos de pruebas y electrodos. Finalmente, se lleva a cabo un análisis en tiempo-frecuencia de las señales EEG con el fin de obtener los parámetros que se correlacionarán con las actividades creativas realizadas.

\subsection{Pretratamiento y filtrado de las señales}

En primer lugar, es necesario llevar a cabo un filtrado de los datos para aislar de mejor forma la información adquirida por cada canal.

\subsubsection{Filtros por hardware}

Parte de los filtros son realizados mediante hardware directamente por el equipo actiCHamp. Concretamente se puede asignar tanto un filtro paso alto, como paso bajo, como notch desde el software Brain Vision. Para esta investigación se asignó un filtro paso alto de $0.5 \mathrm{~Hz}$ con el fin de eliminar la componente de continua, un filtro paso bajo de $100 \mathrm{~Hz}$ para eliminar el ruido de medida y las componentes de alta frecuencia con poca información, y un filtro notch a $50 \mathrm{~Hz}$ para mitigar la componente asociada a la interferencia de la red.

\subsubsection{Filtros por software}

Una vez la señal ha sido registrada, resulta conveniente aplicar un filtro espacial con el fin de minimizar la contribución del resto de electrodos a cada canal. De este modo es posible aislar la información de cada sensor mejor [11]. El filtro espacial utilizado fue el Laplaciano. La ecuación 1 muestra cómo se aplica.

$$
V_{i}^{L p}=V_{i}-\sum_{i \neq j} g_{i j} \cdot V_{j}
$$

Siendo $V_{i}^{L p}$ la tensión en el electrodo $i$ tras realizar el filtro Laplaciano, $V_{j}$ la tensión previa en el electrodo

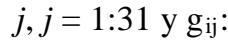

$$
g_{i j}=\frac{\frac{1}{d_{i j}}}{\sum_{i \neq j \neq j} \frac{1}{d_{i j}}}
$$

Representando $\mathrm{d}_{\mathrm{ij}}$ la distancia euclidea entre los electrodos $i \mathrm{y} j$. 
El segundo filtro por software intenta mitigar los artefactos de movimiento ajenos a la actividad mental del usuario. Con este fin, se utilizó la transformada de wavelet mediante una función madre de aproximación discreta de Meyer [10]. Realizando la descomposición a 6 niveles de detalle y eliminando la aproximación reconstruida de nivel 6. La figura 6 muestra una señal con claros artefactos debido a parpadeos de un sujeto de pruebas.
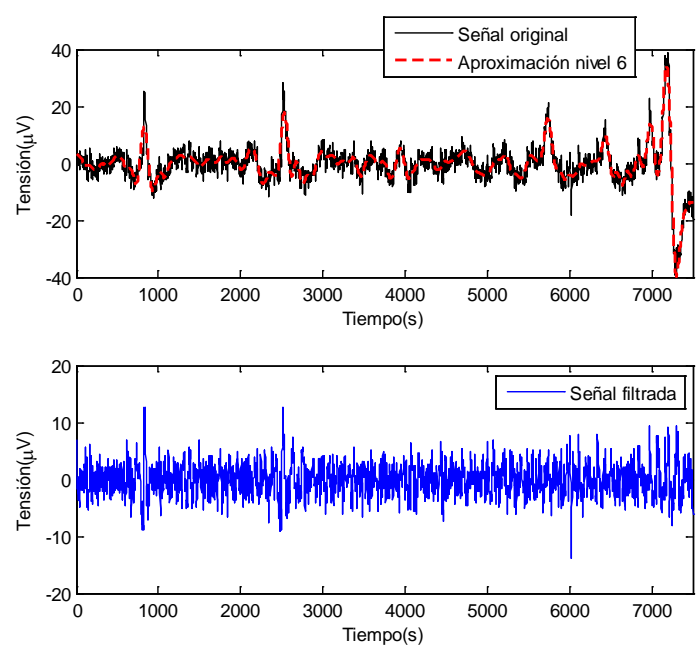

Figura 6: Filtrado de artefactos con wavelet mediante aproximación discreta de Meyer. El ejemplo muestra

el filtrado de un sujeto en la prueba P1 para el electrodo Fz.

\subsection{Normalización de las señales EEG}

Al contrario que en otras investigaciones previas realizadas [12,13], donde las señales se utilizan para generar un modelo independiente por sujeto de pruebas, esta investigación requiere de una comparación de los parámetros extraídos entre sujetos. Por ello, es necesario llevar a cabo una normalización que nos permita comparar en una misma escala los datos extraídos. Adicionalmente, y debido a que, en base al estado del arte, no se puede sacar una conclusión previa clara de las zonas cerebrales que aportan mayor información durante el proceso de pensamiento creativo-artístico, resulta preciso llevar a cabo una normalización de los datos utilizando los 31 canales registrados.

La normalización utilizada se basa en el máximo umbral visual (MV, en Inglés Maximum Visual Threshold) introducida en [2]. El MV umbral se puede obtener para la tensión $V$ de un electrodo $i$ mediante la ecuación 3:

$M V^{i}=\frac{1}{N} \sum_{m=1}^{N} \max \left[a b s\left(V_{(m-1) \cdot L+1: m \cdot L}^{i}\right)\right]$

Siendo $\mathrm{N}$ el número de subventanas a considerar por prueba, tomándose una subventana de 500 muestras
(1 segundo) cada $\mathrm{L}=100$ muestras (0.2 segundos). Una vez se tiene el MV umbral para cada electrodo, se computa el valor normalizado de tensión del electrodo $i$ para todo instante $t$ como:

$$
S V^{i}(t)=\frac{V^{i}(t)}{\frac{1}{31} \cdot \sum_{j=1}^{31} M V^{j}}
$$

De este modo, al promediar los umbrales MV de los 31 canales no se pierde información en la normalización y se puede realizar una comparación entre sujetos y canales en un mismo orden de magnitud.

\subsection{Análisis de las señales EEG}

Una vez las señales han sido filtradas y normalizadas por sujeto de pruebas, se calcula la densidad espectral de potencia mediante el método de Burg. La figura 7 muestra un ejemplo de análisis para el electrodo $\mathrm{Fz}$ en base a los datos normalizados de tensión según el punto anterior.
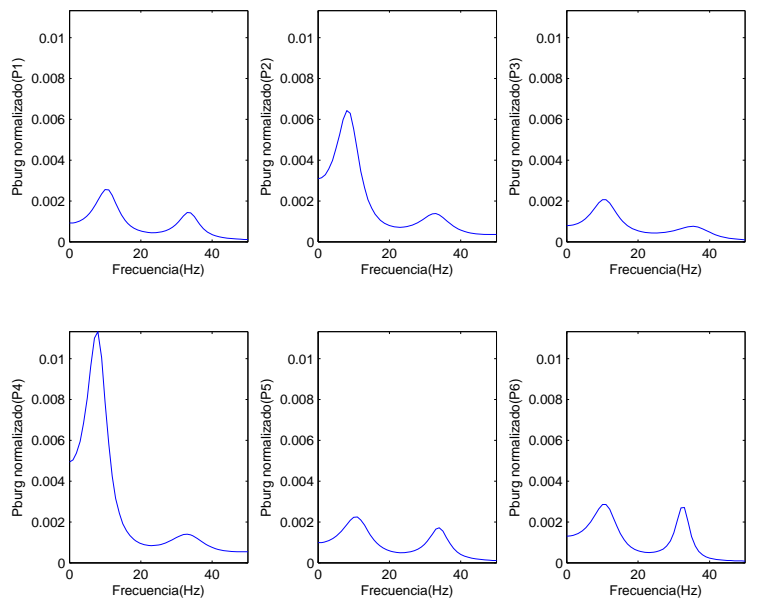

Figura 7: Densidad espectral normalizada de un sujeto para las pruebas P1-P6. El ejemplo muestra la información extraída del electrodo Fz.

\section{RESULTADOS}

Como se aprecia en la figura 7. La densidad de potencia espectral muestra dos aumentos en la señal EEG en el caso particular del electrodo Fz. Uno alrededor de la banda alfa y otro en baja gamma. El aumento de la banda alfa se da especialmente en las acciones pictóricas debido al aumento de la señal cerebral durante la acción motora (pruebas P2 y P4). La banda gamma experimenta cambios más leves a lo largo de las pruebas para dicho electrodo. Aunque se aprecia un aumento de la banda gamma en relax (P6) con respecto a las actividades de imaginación creativa (P1 y P3), o contemplativa de una obra pictórica (P5), resulta difícil extraer conclusiones basadas en un solo sujeto y electrodo. 
 TRABAJOS}

En el trabajo de este artículo se ha mostrado un protocolo de pruebas y metodología de análisis para la medición de la actividad cerebral mediante señales EEG durante actividades creativo-artísticas.

El siguiente paso en la investigación debe basarse en diversos puntos. En primer lugar, identificar qué grupo de electrodos experimenta una mayor variación durante las pruebas de forma consistente en un grupo de sujetos de pruebas. En segundo lugar, identificar, dentro de esos electrodos, qué bandas son las que tienen una mayor variación porcentual. El tercer punto debe intentar buscar una correlación entre las variaciones observadas entre sujetos y las obras pictóricas realizadas, con el fin de poder contrastar las diferencias en EEG con respecto a la creatividad plasmada en las láminas.

\section{Agradecimientos}

El sistema inalámbrico de registro de señales EEG de 32 canales de Brain Products ha sido cofinanciado con fondos procedentes de la Unión Europea (P.O. FEDER 2007/2013), con la gestión de la Generalitat Valenciana.

\section{English summary}

\section{A PRELIMINARY STUDY OF THE EVALUATION OF ARTISTIC CREATIVITY BASED ON THE ANALYSIS OF ELECTROENCEPHALOGRAPHIC SIGNALS}

\footnotetext{
Abstract

Creativity is usually evaluated by creativity tests, such as Torrance creativity thinking test. This test is extended in education and companies. However, because of the characteristics of the test, it is necessary the participation and supervision of experts in the evaluation of the performed tasks, adding a subjective point to the qualification. Our research tries to set up the basis to an alternative evaluation of creativity, based on the analysis of the electroencephalographic signals of a subject during a process of creativity thinking and action. The article shows the protocols proposed and the selected signal processing algorithms applied to the electroencephalographic signals acquired. All of this, with the future intention of obtaining an objective index of creativity.
}

Keywords: Brain-Computer Interface, creativity test, electroencephalographic signals, creativity, timefrequency analysis.

\section{Referencias}

[1]. Beudt, S., \& Jacobsen, T. (2015). On the Role of Mentalizing Processes in Aesthetic Appreciation: An ERP Study. Frontiers in Human Neuroscience, 9. https://doi.org/10.3389/fnhum.2015.00600

[2]. Costa, Á., Iáñez, E., Úbeda, A., Hortal, E., Del-Ama, A. J., Gil-Agudo, Á., \& Azorín, J. M. (2016). Decoding the attentional demands of gait through EEG gamma band features. $\quad P L O S \quad O N E$, 11(4). https://doi.org/10.1371/journal.pone.015413 6

[3]. Dietrich, A., \& Kanso, R. (2010). A review of EEG, ERP, and neuroimaging studies of creativity and insight. Psychological Bulletin, 136(5), 822-848. https://doi.org/10.1037/a0019749

[4]. Ellamil, M., Dobson, C., Beeman, M., \& Christoff, K. (2012d). Evaluative and generative modes of thought during the creative process. NeuroImage, 59(2), 17831794.

https://doi.org/10.1016/j.neuroimage.2011.0 8.008

[5]. González, J. E. J. (2007). Adaptación y baremación del test de pensamiento creativo de Torrance: expresión figurada. Educación Primaria y Secundaria. Dirección General de Ordenación E Innovación Educativa de La Consejería de Educación, Cultura $Y$ Deportes Del Gobierno de Canarias, Comunidad Autónoma de Canarias, 157.

[6]. Kim, K. H. (2006). Can we trust creativity tests? A review of the Torrance Tests of Creative Thinking (TTCT). Creativity Research Journal, 18(1), 3-14. https://doi.org/10.1207/s15326934crj1801_2

[7]. Kontson, K. L., Megjhani, M., Brantley, J. A., Cruz-Garza, J. G., Nakagome, S., Robleto, D., Contreras-Vidal, J. L. (2015). Your Brain on Art: Emergent Cortical Dynamics During Aesthetic Experiences. Frontiers in Human Neuroscience, 9. https://doi.org/10.3389/fnhum.2015.00626

[8]. Limb, C. J., \& Braun, A. R. (2008). Neural substrates of spontaneous musical performance: An fMRI study of jazz improvisation. PLoS ONE, 3(2), e1679. https://doi.org/10.1371/journal.pone.000167 9 
[9]. Liu, S., Chow, H. M., Xu, Y., Erkkinen, M. G., Swett, K. E., Eagle, M. W., Braun, A. R. (2012). Neural correlates of lyrical improvisation: An fMRI study of freestyle rap. Scientific Reports, 2(1), 834.

https://doi.org/10.1038/srep00834

[10]. Mallat, S. G. (1989). A Theory for Multiresolution Signal Decomposition: The Wavelet Representation. IEEE Transactions on Pattern Analysis and Machine Intelligence, 11(7),674-693.

https://doi.org/10.1109/34.192463

[11]. Mcfarland, D. J., Mccane, L. M., David, S. V, \& Wolpaw, J. R. (1997). Spatial filter selection for EEG - based communication. Electroencephalography and Clinical Neurophysiology, 103(3), 386-394.

https://doi.org/10.1016/S00134694(97)00022-2

[12]. Ortiz, M., Rodríguez-Ugarte, M., Iáñez, E., \& Azorín, J. M. (2017). Application of the Stockwell Transform to Electroencephalographic Signal Analysis during Gait Cycle. Frontiers in Neuroscience, 11, 660.

https://doi.org/10.3389/fnins.2017.00660

[13]. Rodríguez-Ugarte, M., Iáñez, E., Ortíz, M., \& Azorín, J. M. (2017). Personalized Offline and Pseudo-Online BCI Models to Detect Pedaling Intent. Frontiers in Neuroinformatics, 11.

https://doi.org/10.3389/fninf.2017.00045

[14]. The Alberta Tearchers' Association. (2007). Torrance Tests of Creative Thinking (TTCT): Figural \& Verbal. 2014 (Vol. 6). Scholastic Testing Service.

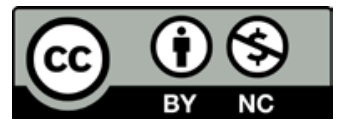

(C) 2018 by the authors. Submitted for possible open access publication under the terms and conditions of the Creative Commons Attribution CC-BY-NC 3.0 license (https://creativecommons.org/licenses/by-nc/3.0). 